Pure and Applied Mathematics Quarterly

Volume 8, Number 1

(Special Issue: In honor of

F. Thomas Farrell and Lowell E. Jones, Part 1 of 2)

$199-219,2012$

\title{
Induction and Computation of Bass Nil Groups for Finite Groups
}

\author{
Ian Hambleton and Wolfgang Lück
}

\begin{abstract}
Let $G$ be a finite group. We show that the Bass Nil-groups $\mathrm{NK}_{n}(R G), n \in \mathbf{Z}$, are generated from the $p$-subgroups of $G$ by induction, certain twistings maps depending on elements in the centralizers of the $p$ subgroups, and the Verschiebung homomorphisms. As a consequence, the groups $\mathrm{NK}_{n}(R G)$ are generated by induction from elementary subgroups. For $\mathrm{NK}_{0}(\mathbf{Z} G)$ we get an improved estimate of the torsion exponent.
\end{abstract}

Keywords: algebraic K-theory, Dress induction, Bass Nil groups.

\section{INTRODUCTION}

In this note we study the Bass Nil-groups (see [2, Chap. XII])

$$
\mathrm{NK}_{n}(R G)=\operatorname{ker}\left(K_{n}(R G[t]) \rightarrow K_{n}(R G)\right),
$$

where $R$ is an associative ring with unit, $G$ is a finite group, $n \in \mathbf{Z}$, and the augmentation map sends $t \mapsto 0$. Alternately, the isomorphism

$$
\mathrm{NK}_{n}(R G) \cong \widetilde{K}_{n-1}(\mathrm{NIL}(R G))
$$

identifies the Bass Nil-groups with the $K$-theory of the category $\operatorname{NIL}(R G)$ of nilpotent endomorphisms $(Q, f)$ on finitely-generated projective $R G$-modules [2,

Received July 5, 2007.

Research partially supported by NSERC Discovery Grant A4000 and by the Max Planck Forschungspreis of the second author. The first author would like to thank the SFB 478, Universität Münster, for its hospitality and support. 
Chap. XII], [13, Theorem 2]. Farrell [8] proved that $\mathrm{NK}_{1}(R G)$ is not finitelygenerated as an abelian group whenever it is non-zero, and the corresponding result holds for $\mathrm{NK}_{n}(R G), n \in \mathbf{Z}$ (see [27, 4.1]), so some organizing principle is needed to better understand the structure of the Nil-groups. Our approach is via induction theory. The functors $\mathrm{NK}_{n}$ are Mackey functors on the subgroups of $G$, and we ask to what extent they can be computed from the Nil-groups of proper subgroups of $G$.

The Bass-Heller-Swan formula [2, Chap. XII, §7], [12, p. 236] relates the Bass Nil-groups with the $K$-theory of the infinite group $G \times \mathbf{Z}$. There are two (split) surjective maps

$$
N_{ \pm}: K_{n}(R[G \times \mathbf{Z}]) \rightarrow \mathrm{NK}_{n}(R G)
$$

which form part of the Bass-Heller-Swan direct sum decomposition

$$
K_{n}(R[G \times \mathbf{Z}])=K_{n}(R G) \oplus K_{n-1}(R G) \oplus \mathrm{NK}_{n}(R G) \oplus \mathrm{NK}_{n}(R G) .
$$

Notice that both $K_{n}(R[(-) \times \mathbf{Z}])$ and $\mathrm{NK}_{n}(R[-])$ are Mackey functors on the subgroups of $G$ (see Farrell-Hsiang [9, §2] for this observation about infinite groups). We observe that the maps $N_{ \pm}$are actually natural transformations of Mackey functors (see Section 6). It follows from Dress induction [7] that the functors $\mathrm{NK}_{n}(R G)$ and the maps $N_{ \pm}$are computable from the hyperelementary family (see Section 4, and Harmon [17, Cor. 4] for the case $n=1$ ). We will show how the results of Farrell [8] and techniques of Farrell-Hsiang [10] lead to a better generation statement for the Bass Nil-groups.

We need some notation to state the main result. For each prime $p$, we denote by $\mathfrak{P}_{p}(G)$ the set of finite $p$-subgroups of $G$, and by $\mathfrak{E}_{p}(G)$ the set of $p$-elementary subgroups of $G$. Recall that a $p$-elementary group has the form $E=C \times P$, where $P$ is a finite $p$-group, and $C$ is a finite cyclic group of order prime to $p$. For each element $g \in C$, we let

$$
I(g)=\{k \in \mathbb{N} \mid \text { a prime } q \text { divides } k \Rightarrow q \text { divides }|g|\}
$$

where $|g|$ denotes the order of $g$. For each $P \in \mathfrak{P}_{p}(G)$, let

$$
C_{G}^{\perp}(P)=\{g \in G \mid g x=x g, \forall x \in P, \text { and } p \nmid|g|\}
$$

and for each $g \in C_{G}^{\perp}(P)$ we define a functor

$$
\phi(P, g): \mathrm{NIL}(R P) \rightarrow \mathrm{NIL}(R G)
$$


by sending a nilpotent $R P$-endomorphism $f: Q \rightarrow Q$ of a finitely-generated projective $R P$-module $Q$ to the nilpotent $R G$-endomorphism

$$
R G \otimes_{R P} Q \rightarrow R G \otimes_{R P} Q, \quad x \otimes q \mapsto x g \otimes f(q) .
$$

Note that this $R G$-endomorphism is well-defined since $g \in C_{G}^{\perp}(P)$. The functor $\phi(P, g)$ induces a homomorphism

$$
\phi(P, g): \mathrm{NK}_{n}(R P) \rightarrow \mathrm{NK}_{n}(R G)
$$

for each $n \in \mathbf{Z}$. For each $p$-subgroup $P$ in $G$, define a homomorphism

$$
\Phi_{P}: \mathrm{NK}_{n}(R P) \rightarrow \mathrm{NK}_{n}(R G)
$$

by the formula

$$
\Phi_{P}=\sum_{g \in C_{G}^{\perp}(P), k \in I(g)} V_{k} \circ \phi(P, g)
$$

where

$$
V_{k}: \mathrm{NK}_{n}(R G) \rightarrow \mathrm{NK}_{n}(R G)
$$

denotes the Verschiebung homomorphism, $k \geq 1$, recalled in more detail in Section 2 .

Theorem A. Let $R$ be an associative ring with unit, and $G$ be a finite group. For each prime $p$, the map

$$
\Phi=\left(\Phi_{P}\right): \bigoplus_{P \in \mathfrak{P}_{p}(G)} \mathrm{NK}_{n}(R P)_{(p)} \rightarrow \mathrm{NK}_{n}(R G)_{(p)}
$$

is surjective for all $n \in \mathbf{Z}$, after localizing at $p$.

For every $g \in C_{G}^{\perp}(P)$, the homomorphism $\phi(P, g)$ factorizes as

$$
\mathrm{NK}_{n}(R P) \stackrel{\phi(P, g)}{\longrightarrow} \mathrm{NK}_{n}(R[C \times P]) \stackrel{i_{*}}{\longrightarrow} \mathrm{NK}_{n}(R G)
$$

where $C=\langle g\rangle$ and $i: C \times P \rightarrow G$ is the inclusion map. Since the Verschiebung homomorphisms are natural with respect to the maps induced by group homomorphisms, we obtain:

Corollary B. The sum of the induction maps

$$
\bigoplus_{E \in \mathfrak{E}_{p}(G)} \mathrm{NK}_{n}(R E)_{(p)} \rightarrow \mathrm{NK}_{n}(R G)_{(p)}
$$

from p-elementary subgroups is surjective, for all $n \in \mathbf{Z}$. 
Note that Theorem A does not show that $\mathrm{NK}_{n}(R G)$ is generated by induction from $p$-groups, because the maps $\phi(P, g)$ for $g \neq 1$ are not the usual maps induced by inclusion $P \subset G$.

The Bass Nil-groups are non-finitely generated torsion groups (if non-zero) so they remain difficult to calculate explicitly, but we have some new qualitative results. For example, Theorem A shows that the order of every element of $\mathrm{NK}_{n}(R G)$ is some power of $m=|G|$, whenever $\mathrm{NK}_{n}(R)=0$ (since its $q$ localization is zero for all $q \nmid m)$. For $R=\mathbf{Z}$ and some related rings, this is a result of Weibel [27, (6.5), p. 490]. In particular, we know that every element of $\mathrm{NK}_{n}(\mathbf{Z} P)$ has $p$-primary order, for every finite $p$-group $P$. If $R$ is a regular (noetherian) ring (e.g. $R=\mathbf{Z}$ ), then $\operatorname{NK}_{n}(R)=0$ for all $n \in \mathbf{Z}$.

Note that an exponent that holds uniformly for all elements in $\mathrm{NK}_{n}(R P)$, over all $p$-groups of $G$, will be an exponent for $\mathrm{NK}_{n}(R G)$. As a special case, we have $\mathrm{NK}_{n}(\mathbf{Z}[\mathbf{Z} / p])=0$ for $n \leq 1$, for $p$ a prime (see Bass-Murthy [4]), so Theorem A implies:

Corollary 1.1. Let $G$ be a finite group and let $p$ be a prime. Suppose that $p^{2}$ does not divide the order of $G$. Then

$$
\mathrm{NK}_{n}(\mathbf{Z} G)_{(p)}=0
$$

for $n \leq 1$.

As an application, we get a new proof of the fact that $\mathrm{NK}_{n}(\mathbf{Z} G)=0$, for $n \leq 1$, if the order of $G$ is square-free (see Harmon [17]).

We also get from Theorem A an improved estimate on the exponent of $\mathrm{NK}_{0}(\mathbf{Z} G)$, using a result of Connolly-da Silva [5]. If $n$ is a positive integer, and $n_{q}=q^{k}$ is its $q$-primary part, then let $c_{q}(n)=q^{l}$, where $l \geq \log _{q}(k n)$. According to [5], the exponent of $\mathrm{NK}_{0}(\mathbf{Z} G)$ divides

$$
c(n)=\prod_{q \mid n} c_{q}(n), \quad \text { where } n=|G|,
$$

but according to Theorem $\mathrm{A}$, the exponent of $\mathrm{NK}_{0}(\mathbf{Z} G)$ divides

$$
d(n)=\prod_{q \mid n} c\left(n_{q}\right) .
$$

For example, $c(60)=1296000$, but $d(60)=120$. 
Acknowledgement: This paper is dedicated to Tom Farrell and Lowell Jones, whose work in geometric topology has been a constant inspiration. We are also indebted to Frank Quinn, who suggested that the Farrell-Hsiang induction techniques should be re-examined (see [20]).

\section{BASS Nil-GroupS}

Standard constructions in algebraic $K$-theory for exact categories or Waldhausen categories yield only $K$-groups in degrees $n \geq 0$ (see Quillen [19], Waldhausen [26]). One approach on the categorial level to negative $K$-groups has been developed by Pedersen-Weibel (see [18], [15, §2.1]). Another ring theoretic approach is given as follows (see Bartels-Lück [1, Section 9], Wagoner [25]). The cone ring $\Lambda \mathbf{Z}$ of $\mathbf{Z}$ is the ring of column and row finite $\mathbb{N} \times \mathbb{N}$-matrices over $\mathbf{Z}$, i.e., matrices such that every column and every row contains only finitely many non-zero entries. The suspension ring $\Sigma \mathbf{Z}$ is the quotient of $\Lambda \mathbf{Z}$ by the ideal of finite matrices. For an associative (but not necessarily commutative) ring $A$ with unit, we define $\Lambda A=\Lambda \mathbf{Z} \otimes_{\mathbf{Z}} A$ and $\Sigma A=\Sigma \mathbf{Z} \otimes_{\mathbf{Z}} A$. Obviously $\Lambda$ and $\Sigma$ are functors from the category of rings to itself. There are identifications, natural in $A$,

$$
\begin{aligned}
K_{n-1}(A) & =K_{n}(\Sigma A) \\
\mathrm{NK}_{n-1}(A) & =\mathrm{NK}_{n}(\Sigma A)
\end{aligned}
$$

for all $n \in \mathbf{Z}$. In our applications, usually $A=R G$ where $R$ is a ring with unit and $G$ is a finite group.

Using these identifications it is clear how to extend the definitions of certain maps between $K$-groups given by exact functors to negative degrees. Moreover, we will explain constructions and proofs of the commutativity of certain diagrams only for $n \geq 1$, and will not explicitly mention that these carry over to all $n \in \mathbf{Z}$, because of the identifications (2.1) and (2.2) and the obvious identification $\Sigma(R G)=(\Sigma R) G$, or because of Pedersen-Weibel [18].

We have a direct sum decomposition

$$
K_{n}(A[t])=K_{n}(A) \oplus \mathrm{NK}_{n}(A)
$$

which is natural in $A$, using the inclusion $A \rightarrow A[t]$ and the ring map $A[t] \rightarrow A$ defined by $t \mapsto 0$. 
Let $\mathcal{P}(A)$ be the exact category of finitely generated projective $A$-modules, and let $\operatorname{NIL}(A)$ be the exact category of nilpotent endomorphism of finitely generated projective $A$-modules. The functor $\mathcal{P}(A) \rightarrow \operatorname{NIL}(A)$ sending $Q \mapsto(Q, 0)$ and the functor $\operatorname{NIL}(A) \rightarrow \mathcal{P}(A)$ sending $(Q, f) \mapsto Q$ are exact functors. They yield a split injection on the $K$-groups $K_{n}(A):=K_{n}(\mathcal{P}(A)) \rightarrow K_{n}(\mathrm{NIL}(A))$ for $n \in \mathbf{Z}$. Denote by $\widetilde{K}_{n}(\mathrm{NIL}(A))$ the cokernel for $n \in \mathbf{Z}$. There is an identification (see Grayson [13, Theorem 2])

$$
\widetilde{K}_{n-1}(\mathrm{NIL}(A))=\mathrm{NK}_{n}(A),
$$

for $n \in \mathbf{Z}$, essentially given by the passage from a nilpotent $A$-endomorphism $(Q, f)$ to the $A \mathbf{Z}$-automorphism

$$
A \mathbf{Z} \otimes_{A} Q \rightarrow A \mathbf{Z} \otimes_{A} Q, \quad u \otimes q \mapsto u \otimes q-u t \otimes f(q),
$$

for $t \in \mathbf{Z}$ a fixed generator.

The Bass Nil-groups appear in the Bass-Heller-Swan decomposition for $n \in \mathbf{Z}$ (see [2, Chapter XII], [3], [12, p. 236], [19, p. 38], and [24, Theorem 10.1] for the original sources, or the expositions in [21, Theorems 3.3.3 and 5.3.30], [22, Theorem 9.8] ).

$$
B: K_{n}(A) \oplus K_{n-1}(A) \oplus \mathrm{NK}_{n}(A) \oplus \mathrm{NK}_{n}(A) \stackrel{\cong}{\rightrightarrows} K_{n}(A \mathbf{Z}) .
$$

The isomorphism $B$ is natural in $A$ and comes from the localization sequence

$$
\begin{aligned}
& 0 \rightarrow K_{n}(A) \stackrel{K_{n}(i) \oplus-K_{n}(i)}{\longrightarrow} K_{n}(A[t]) \oplus K_{n}(A[t]) \stackrel{K_{n}\left(j_{+}\right)+K_{n}\left(j_{-}\right)}{\longrightarrow} K_{n}(A \mathbf{Z}) \\
& \stackrel{\partial_{n}}{\longrightarrow} K_{n-1}(A) \rightarrow 0
\end{aligned}
$$

where $i: A \rightarrow A[t]$ is the obvious inclusion and the inclusion $j_{ \pm}: A[t] \rightarrow A \mathbf{Z}$ sends $t$ to $t^{ \pm 1}$ if we write $A \mathbf{Z}=A\left[t, t^{-1}\right]$, the splitting of $\partial_{n}$

$$
s_{n}: K_{n-1}(A) \rightarrow K_{n}(A \mathbf{Z})
$$

which is given by the natural pairing

$$
K_{n-1}(A) \otimes K_{1}\left(\mathbf{Z}\left[t, t^{-1}\right]\right) \rightarrow K_{n}\left(A \otimes_{\mathbf{Z}} \mathbf{Z}\left[t, t^{-1}\right]\right)=K_{n}(A \mathbf{Z})
$$

evaluated at the class of unit $t \in \mathbf{Z}\left[t, t^{-1}\right]$ in $K_{1}\left(\mathbf{Z}\left[t, t^{-1}\right]\right)$, and the canonical splitting (2.3). Let $B$ be the direct sum of $K_{n}\left(j_{+} \circ i\right), s_{n}$, and the restrictions of the maps $K_{n}\left(j_{+}\right)$and $K_{n}\left(j_{-}\right)$to $\mathrm{NK}_{n}(A)$. 
In particular we get two homomorphisms, both natural in $A$, from the BassHeller-Swan decomposition (2.5)

$$
\begin{aligned}
& i_{n}: \mathrm{NK}_{n}(A) \rightarrow K_{n}(A \mathbf{Z}) \\
& r_{n}: K_{n}(A \mathbf{Z}) \rightarrow \mathrm{NK}_{n}(A),
\end{aligned}
$$

by focusing on the first copy of $\mathrm{NK}_{n}(A)$, such that $r_{n} \circ i_{n}$ is the identity on $\mathrm{NK}_{n}(A)$.

Let $\sigma_{k}: \mathbf{Z} \rightarrow \mathbf{Z}$ be the injection given by $t \mapsto t^{k}$. We may consider the ring $A[t]$ as an $A[t]-A[t]$ bimodule with standard left action, and right action $a(t) \cdot b(t)=a(t) b\left(t^{k}\right)$ induced by $\sigma_{k}$. This map induces an induction functor

$$
\operatorname{Ind}_{k}: \mathcal{P}(A[t]) \rightarrow \mathcal{P}(A[t])
$$

defined by $P \mapsto A[t] \otimes_{\sigma_{k}} P$. There is also a restriction functor

$$
\operatorname{Res}_{k}: \mathcal{P}(A[t]) \rightarrow \mathcal{P}(A[t])
$$

defined by equipping $P$ with the new $A[t]$-module structure $a(t) \cdot p=a\left(t^{k}\right) p$, for all $a(t) \in A[t]$ and all $p \in P$. The induction and restriction functors yield two homomorphisms

$$
\begin{aligned}
\operatorname{Ind}_{k}: K_{n}(A \mathbf{Z}) \rightarrow K_{n}(A \mathbf{Z}) \\
\operatorname{Res}_{k}: K_{n}(A \mathbf{Z}) \rightarrow K_{n}(A \mathbf{Z}) .
\end{aligned}
$$

See [23] or [19, p. 27] for more details.

There are also Verschiebung and Frobenius homomorphisms

$$
V_{k}, F_{k}: \mathrm{NK}_{n}(A) \rightarrow \mathrm{NK}_{n}(A)
$$

induced on the Nil-groups (and related to $\operatorname{Ind}_{k}$ and $\operatorname{Res}_{k}$ respectively). The Frobenius homomorphism is induced by the functor $\operatorname{NIL}(A) \rightarrow \operatorname{NIL}(A)$ sending

$$
(f: Q \rightarrow Q) \mapsto\left(f^{k}: Q \rightarrow Q\right),
$$

while the Verschiebung homomorphism is induced by the functor

$$
\bigoplus_{i=1}^{k} Q \rightarrow \bigoplus_{i=1}^{k} Q, \quad\left(q_{1}, q_{2}, \ldots q_{k}\right) \mapsto\left(f\left(q_{k}\right), q_{1}, q_{2}, \ldots, q_{k-1}\right)
$$

The next result is proven by Stienstra [23, Theorem 4.7]). (Notice that Stienstra considers only commutative rings $A$, but his argument goes through in our 
case since the set of polynomials $T$ we consider is $\left\{t^{n} \mid n \in \mathbf{Z}, n \geq 0\right\}$ and each polynomial in $T$ is central in $A[t]$ with respect to the multiplicative structure.)

Lemma 2.9. The following diagrams commute for all $n \in \mathbf{Z}$ and $k \in \mathbf{Z}, k \geq 1$



and



The next result is well-known for $n=1$ (see Farrell [8, Lemma 3]). The general case is discussed by Weibel [27, p. 479], Stienstra [23, p. 90], and Grunewald [11, Prop. 4.6].

Lemma 2.10. For every $n \in \mathbf{Z}$ and each $x \in \mathrm{NK}_{n}(A)$, there exists a positive integer $M(x)$ such that $\operatorname{Res}_{m} \circ i_{n}(x)=0$ for $m \geq M(x)$.

Proof. The Frobenius homomorphism $F_{m}: \mathrm{NK}_{n}(A) \rightarrow \mathrm{NK}_{n}(A)$ is induced by the functor sending $(Q, f)$ in $\operatorname{NIL}(A)$ to $\left(Q, f^{m}\right)$. For a given object $(Q, f)$ in $\operatorname{NIL}(A)$ there exists a positive integer $M(f)$ with $\left(Q, f^{m}\right)=(Q, 0)$ for $m \geq M(f)$. This implies by a filtration argument (see [23, p. 90] or [11, Prop. 4.6]), that for $x \in \mathrm{NK}_{n}(A)$ there exists a positive integer $M(x)$ with $F_{m}(x)=0$ for $m \geq M(x)$. Now the claim follows from Lemma 2.9 .

\section{Subgroups of $G \times \mathbf{Z}$}

A finite group $G$ is called $p$-hyperelementary if is isomorphic to an extension

$$
1 \rightarrow C \rightarrow G \rightarrow P \rightarrow 1
$$

where $P$ is a $p$-group, and $C$ is a cyclic group of order prime to $p$. Such an extension is a semi-direct product, and hence determined by the action map $\alpha: P \rightarrow \operatorname{Aut}(C)$ defined by conjugation. The group $G$ is $p$-elementary precisely when $\alpha$ is the trivial map, or in other words, when there exists a retraction 
$G \rightarrow C$. Notice that for a cyclic group $C=\mathbf{Z} / q^{k}$, where $q \neq p$ is a prime, $\operatorname{Aut}(C)=\mathbf{Z} / q^{k-1}(q-1)$, if $q$ odd, or $\operatorname{Aut}(C)=\mathbf{Z} / 2^{k-2} \times \mathbf{Z} / 2, k \geq 2$, if $q=2$. In either case, $\operatorname{Aut}(C)_{(p)} \cong \operatorname{Aut}(Q)_{(p)}$ by projection to any non-trivial quotient group $C \rightarrow Q$.

Lemma 3.1. Let $p$ be a prime, and let $G$ be a finite p-hyperelementary group. Suppose that for every prime $q \neq p$ which divides the order of $G$, there exists an epimorphism $f_{q}: G \rightarrow Q_{q}$ onto a non-trivial cyclic group $Q_{q}$ of $q$-power order. Then $G$ is p-elementary.

Proof. Let $Q$ be the product of the groups $Q_{q}$ over all primes $q \neq p$ which divide the order of $G$. Let $f: G \rightarrow Q$ be the product of the given epimorphisms. Since every subgroup in $G$ of order prime to $p$ is characteristic, we have a diagram

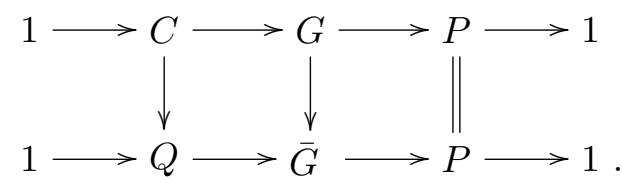

But the epimorphism $f: G \rightarrow Q$ induces a retraction $\bar{G} \rightarrow Q$ of the lower sequence, hence its action map $\bar{\alpha}: P \rightarrow \operatorname{Aut}(Q)$ is trivial. As remarked above, this implies that $\alpha$ is also trivial and hence $G$ is $p$-elementary.

We now combine this result with the techniques of [10]. Given positive integers $m, n$ and a prime $p$, we choose an integer $N=N(m, n, p)$ satisfying the following conditions:

(i) $p \nmid N$, but $q \mid N$ if and only if $q \mid n$ for all primes $q \neq p$.

(ii) $k \geq \log _{q}(m n)$ (i.e. $q^{k} \geq m n$ ) for each full prime power $q^{k} \| N$.

The Farrell-Hsiang technique is to compute $K$-theory via $p$-hyperelementary subgroups $H \subset G \times \mathbf{Z} / N$, and their inverse images $\Gamma_{H}=\operatorname{pr}^{-1}(H) \subset G \times \mathbf{Z}$ via the second factor projection map pr: $G \times \mathbf{Z} \rightarrow G \times \mathbf{Z} / N$.

Lemma 3.2. Let $G$ be a finite group and let $M$ be a positive integer. Let $p$ be a prime dividing the order of $|G|$, and choose an integer $N=N(M,|G|, p)$. For every p-hyperelementary subgroup $H \subset G \times \mathbf{Z} / N$, one of the following holds:

(i) the inverse image $\Gamma_{H} \subset G \times m \cdot \mathbf{Z}$, for some $m \geq M$, or

(ii) the group $H$ is p-elementary. 
In the second case, we have the following additional properties:

(a) There exists a finite p-group $P$ and isomorphism

$$
\alpha: P \times \mathbf{Z} \stackrel{\cong}{\longrightarrow} \Gamma_{H}
$$

(b) There exists a positive integer $k$, a positive integer $\ell$ with $(\ell, p)=1$, an element $u \in \mathbf{Z} / \ell$ and an injective group homomorphism

$$
j: P \times \mathbf{Z} / \ell \rightarrow G
$$

such that the following diagram commutes



where $i: \Gamma_{H} \rightarrow G \times \mathbf{Z}$ is the inclusion and $\beta: \mathbf{Z} \rightarrow \mathbf{Z} / \ell \times \mathbf{Z}$ sends $n$ to $(n u, n)$.

Proof. In the proof we will write elements in $\mathbf{Z} / N$ additively and elements in $G$ multiplicatively. Let $H \subset G \times \mathbf{Z} / N$ be a $p$-hyperelementary subgroup, and suppose that $\Gamma_{H}$ is not contained in $G \times m \cdot \mathbf{Z}$ for any $m \geq M$. We have a pull-back diagram

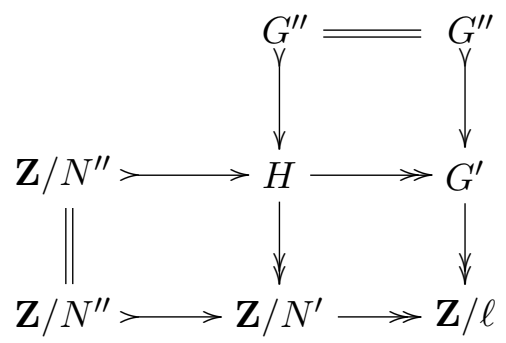

where $G^{\prime} \subset G$ and $\mathbf{Z} / N^{\prime}$ are the images of $H \subset G \times \mathbf{Z}$ under the first and second factor projection, respectively. Notice that $\mathbf{Z} / \ell$ is the common quotient group of $G^{\prime}$ and $\mathbf{Z} / N^{\prime}$. In terms of this data, $H \subseteq G^{\prime} \times \mathbf{Z} / N^{\prime}$ and hence the pre-image $\Gamma_{H} \subseteq G^{\prime} \times m \cdot \mathbf{Z} \subseteq G \times m \cdot \mathbf{Z}$, where $m=N / N^{\prime}$.

We now show that $G^{\prime \prime}$ is a $p$-group. Suppose, if possible, that some other prime $q \neq p$ divides $\left|G^{\prime \prime}\right|$. Since $H$ is $p$-hyperelementary the Sylow $q$-subgroup of $H$ is 
cyclic. However $G^{\prime \prime} \times \mathbf{Z} / N^{\prime \prime} \subseteq H$, so $q \nmid N^{\prime \prime}$. But $N^{\prime}=N^{\prime \prime} \cdot \ell$, hence this implies that the $q$-primary part $N_{q}^{\prime}=\ell_{q} \leq\left|G^{\prime}\right| \leq|G|$. Now

$$
m=N / N^{\prime} \geq q^{k} / N_{q}^{\prime} \geq q^{k} /|G| \geq M
$$

by definition of $N=N(M,|G|, p)$. This would imply that $\Gamma_{H} \subset G \times m \cdot \mathbf{Z}$ for some $m \geq M$, contrary to our assumption. Hence $P:=G^{\prime \prime}$ is a $p$-group, or more precisely the $p$-Sylow subgroup of $G^{\prime}$ since $p \nmid \ell$.

Alternative (ii) is an immediate consequence. If $q \neq p$ is a prime dividing $|H|$, then $q \mid N^{\prime}$ since $G^{\prime \prime}$ is a $p$-group. Hence $H$ admits an epimorphism onto a nontrivial finite cyclic $q$-group. By Lemma 3.1, this implies that $H$ is $p$-elementary. Note that there is an isomorphism

$$
j^{\prime}=\left(i d_{P} \times s\right): P \times \mathbf{Z} / \ell \stackrel{\cong}{\rightrightarrows} G^{\prime}
$$

defined by the inclusion $i d_{P}: P \subset G^{\prime}$ and a splitting $s: \mathbf{Z} / \ell \rightarrow G^{\prime}$ of the projection $G^{\prime} \rightarrow \mathbf{Z} / \ell$.

Next we consider assertion (a). A similar pull-back diagram exists for the subgroup $\Gamma_{H} \subset G \times \mathbf{Z}$. We obtain a pull-back diagram of exact sequences

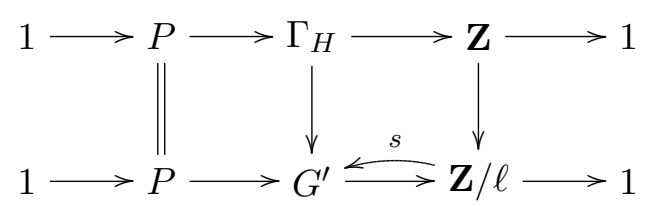

since $P=\Gamma_{H} \cap(G \times 0)$, and $\operatorname{pr}_{\mathbf{Z}}\left(\Gamma_{H}\right)=k \cdot \mathbf{Z}$ for some positive integer $k$. This exact sequence splits, since it is the pull-back of the lower split sequence: we can choose the element $\left(g_{0}, k\right) \in \Gamma_{H} \subseteq G^{\prime} \times \mathbf{Z}$ which projects to a generator of $\operatorname{pr}_{\mathbf{Z}}\left(\Gamma_{H}\right)$, by taking $g_{0}=s(u)$ where $u \in \mathbf{Z} / \ell$ is a generator. The isomorphism $\alpha: P \times \mathbf{Z} \stackrel{\approx}{\rightarrow} \Gamma_{H}$ is defined by $\alpha(g, n)=\left(g g_{0}^{n}, n\right)$ for $g \in P$ and $n \in \mathbf{Z}$.

Assertion (b) follows by composing the splitting $\left(i d_{P} \times s\right): P \times \mathbf{Z} / \ell \cong G^{\prime}$ with the inclusion $G^{\prime} \subseteq G$ to obtain an injection $j: P \times \mathbf{Z} / \ell \rightarrow G$. By the definition of $g_{0}$, the composite $\left(j \times k \cdot i d_{\mathbf{Z}}\right) \circ\left(i d_{P} \times \beta\right)=i \circ \alpha$, where $i: \Gamma_{H} \rightarrow G \times \mathbf{Z}$ is the inclusion.

\section{The Proof of Theorem A}

We will need some standard results from induction theory for Mackey functors over finite groups, due to Dress (see [6], [7]), as well as a refinement called the 
Burnside quotient Green ring associated to a Mackey functor (see [14, §1] for a description of this construction, and [16] for the detailed account).

For any homomorphism pr: $\Gamma \rightarrow G$ from an infinite discrete group to a finite group $G$, the functor

$$
\mathcal{M}(H):=K_{n}\left(R \Gamma_{H}\right)
$$

where $\Gamma_{H}=\operatorname{pr}^{-1}(H)$, is a Mackey functor defined on subgroups $H \subseteq G$. The required restriction maps exist because the index $\left[\Gamma_{H}: \Gamma_{K}\right]$ is finite for any pair of subgroups $K \subset H$ in $G$. This point of view is due to Farrell and Hsiang [9, $§ 2]$. The Swan ring $S W(G, \mathbf{Z})$ acts as a Green ring on $\mathcal{M}$, and it is a fundamental fact of Dress induction theory that the Swan ring is computable from the family $\mathcal{H}$ of hyperelementary subgroups of $G$. More precisely, the localized Green ring $S W(G, \mathbf{Z})_{(p)}$ is computable from the family $\mathcal{H}_{p}$ of $p$-hyperelementary subgroups of $G$, for every prime $p$. If follows from Dress induction that the Mackey functor $\mathcal{M}(G)_{(p)}$ is also $p$-hyperelementary computable. We need a refinement of this result.

Theorem 4.1 ([14, Theorem 1.8]). Suppose that $\mathcal{G}$ is a Green ring which acts on a Mackey functor $\mathcal{M}$. If $\mathcal{G} \otimes \mathbf{Z}_{(p)}$ is $\mathcal{H}$-computable, then every $x \in \mathcal{M}(G) \otimes \mathbf{Z}_{(p)}$ can be written as

$$
x=\sum_{H \in \mathcal{H}_{p}} a_{H} \operatorname{Ind}_{H}^{G}\left(\operatorname{Res}_{G}^{H}(x)\right)
$$

for some coefficients $a_{H} \in \mathbf{Z}_{(p)}$.

We fix a prime $p$. For each element $x \in \mathrm{NK}_{n}(R G)$, let $M=M(x)$ as in Lemma 2.10 applied to the ring $A=R G$. Then

$$
\operatorname{Res}_{m}: K_{n}(R[G \times \mathbf{Z}]) \rightarrow K_{n}(R[G \times \mathbf{Z}])
$$

sends $i_{n}(x)$ to zero for $m \geq M(x)$. Now let $N=N(M,|G|, p)$, as defined in Section 3, and consider $\mathcal{M}(H)=K_{n}\left(R \Gamma_{H}\right)$ as a Mackey functor on the subgroups $H \subseteq G \times \mathbf{Z} / N$, via the projection $\operatorname{pr}: G \times \mathbf{Z} \rightarrow G \times \mathbf{Z} / N$.

Let $\mathcal{H}_{p}(x)$ denote the set of $p$-hyperelementary subgroups $H \subseteq G \times \mathbf{Z} / N$, such that $\Gamma_{H}$ is not contained in $G \times m \cdot \mathbf{Z}$, for any $m \geq M(x)$. By the formula of Theorem 4.1, applied to $y=i_{n}(x)$, we see that $x$ lies in the image of the composite map

$$
\bigoplus_{H \in \mathcal{H}_{p}(x)} K_{n}\left(R \Gamma_{H}\right)_{(p)} \stackrel{i_{*}}{\rightarrow} K_{n}(R[G \times \mathbf{Z}])_{(p)} \stackrel{r_{n}}{\longrightarrow} \mathrm{NK}_{n}(R G)_{(p)} .
$$


We conclude from Lemma 3.2 (b) (using that notation) that the composite

$$
K_{n}(R[P \times \mathbf{Z}]) \stackrel{\alpha_{*}}{\longrightarrow} K_{n}\left(R \Gamma_{H}\right) \stackrel{i_{*}}{\longrightarrow} K_{n}(R[G \times \mathbf{Z}]) \stackrel{r_{n}}{\longrightarrow} \mathrm{NK}_{n}(R G)
$$

agrees with the composite

$$
\begin{aligned}
& K_{n}(R[P \times \mathbf{Z}]) \stackrel{\left(\operatorname{id}_{P} \times \beta\right)_{*}}{\longrightarrow} K_{n}(R[P \times \mathbf{Z} / \ell \times \mathbf{Z}]) \stackrel{\left(j \times \mathrm{id}_{\mathbf{Z}}\right)_{*}}{\longrightarrow} K_{n}(R[G \times \mathbf{Z}]) \\
& \stackrel{\left(\mathrm{id}_{G} \times k \cdot \mathrm{id}_{\mathbf{z}}\right)_{*}}{\longrightarrow} K_{n}(R[G \times \mathbf{Z}]) \stackrel{r_{n}}{\longrightarrow} \mathrm{NK}_{n}(R G) .
\end{aligned}
$$

Recall that $\beta: \mathbf{Z} \rightarrow \mathbf{Z} / \ell \times \mathbf{Z}$ sends $n$ to $(n u, n)$ for some generator $u \in \mathbf{Z} / \ell$. Let $\operatorname{NIL}(R P) \rightarrow \operatorname{NIL}(R[P \times \mathbf{Z} / \ell])$ be the functor which sends a nilpotent $R G$ endomorphism $f: Q \rightarrow Q$ of a finitely generated $R P$-module $Q$ to the nilpotent $R[G \times \mathbf{Z} / \ell]$-endomorphism

$$
R[P \times \mathbf{Z} / l] \otimes_{R P} Q \mapsto R[P \times \mathbf{Z} / \ell] \otimes_{R P} Q, \quad x \otimes q \mapsto x u \otimes f(q) .
$$

Let $\phi: \mathrm{NK}_{n}(R P) \rightarrow \mathrm{NK}_{n}(R[P \times \mathbf{Z} / \ell])$ denote the induced homomorphism.

\section{Lemma 4.5.}

(a) The following diagram commutes



(b) The following diagram commutes



(c) The following diagram commutes

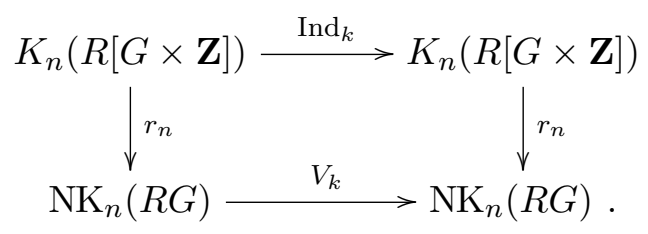


Proof. (a) The tensor product $\otimes \mathbf{z}$ induces a pairing

$$
\mu_{R, \Gamma}: K_{n-1}(R) \otimes_{\mathbf{Z}} K_{1}(\mathbf{Z} \Gamma) \rightarrow K_{n}(R \Gamma)
$$

for every group $\Gamma$, which is natural in $R$ and $\Gamma$. It suffices to prove that the following diagram is commutative for every ring $R$ (since we can replace $R$ by $R P)$. Let $A=R[\mathbf{Z} / \ell]$ for short.

$$
\begin{aligned}
& K_{n}(R) \oplus K_{n-1}(R) \oplus \mathrm{NK}_{n}(R) \oplus \mathrm{NK}_{n}(R) \stackrel{B}{\cong} K_{n}(R \mathbf{Z}) \\
& \left(\begin{array}{cccc}
i_{1,1} & 0 & 0 & 0 \\
i_{1,2} & i_{2,2} & 0 & 0 \\
0 & 0 & \phi & 0 \\
0 & 0 & 0 & \phi
\end{array}\right) \mid \\
& K_{n}(A) \oplus K_{n-1}(A) \stackrel{\mathrm{NK}_{n}(A) \oplus \mathrm{NK}_{n}(A) \stackrel{B}{\cong} K_{n}(A \mathbf{Z})}{\downarrow}
\end{aligned}
$$

Here the vertical arrows are the isomorphisms given by the Bass-Heller-Swan decomposition (2.5), the homomorphisms $i_{1,1}$ and $i_{2,2}$ are induced by the inclusion $R \rightarrow R[\mathbf{Z} / \ell]$ and the homomorphism $i_{1,2}$ comes from the pairing

$$
\mu_{R, \mathbf{Z} / \ell}: K_{n-1}(R) \otimes_{\mathbf{Z}} K_{1}(\mathbf{Z}[\mathbf{Z} / \ell]) \rightarrow K_{n}(R[\mathbf{Z} / \ell])
$$

evaluated at the class of the unit $u \in \mathbf{Z}[\mathbf{Z} / \ell]$ in $K_{1}(\mathbf{Z}[\mathbf{Z} / \ell])$ and the obvious change of rings homomorphisms $K_{1}(R[\mathbf{Z} / \ell]) \rightarrow K_{1}(R[\mathbf{Z} / \ell \times \mathbf{Z}])$

In order to show commutativity it suffices to prove its commutativity after restricting to one of the four summands in the left upper corner.

This is obvious for $K_{n}(R G)$ since induction with respect to group homomorphisms is functorial.

For $K_{n-1}(R)$ this follows from the naturality of the pairing (4.6) in $R$ and the group $\mathbf{Z} / \ell$ and the equality

$$
K_{1}(\beta)(t)=K_{1}\left(R\left[j_{\mathbf{Z}}\right]\right)(t)+K_{1}\left(R\left[j_{\mathbf{Z} / \ell}\right]\right)(u)
$$

where $j_{\mathbf{Z}}: \mathbf{Z} \rightarrow \mathbf{Z} / \ell \times \mathbf{Z}$ and $j_{\mathbf{Z} / \ell}: \mathbf{Z} / \ell \rightarrow \mathbf{Z} / \ell \times \mathbf{Z}$ are the obvious inclusions.

The commutativity when restricted to the two Bass Nil-groups follows from a result of Stienstra [23, Theorem 4.12 on page 78].

(b) This follows from the naturality in $R$ of $r_{n}$. 
(c) It suffices to show that the following diagram commutes (since we can replace $R$ by $R G)$

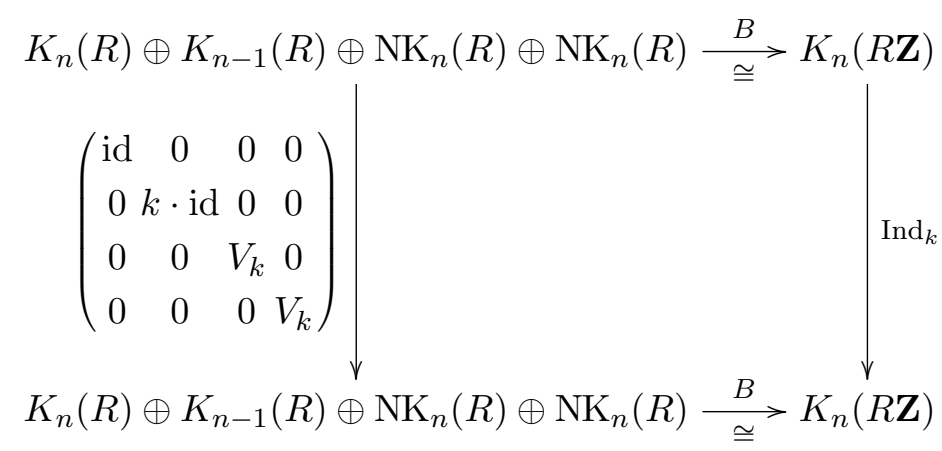

where the vertical arrows are the isomorphisms given by the Bass-Heller-Swan decomposition (2.5).

In order to show commutativity it suffices to prove its commutativity after restricting to one of the four summands in the left upper corner.

This is obvious for $K_{n}(R)$ since induction with respect to group homomorphisms is functorial.

Next we inspect $K_{n-1}(R)$. The following diagram commutes

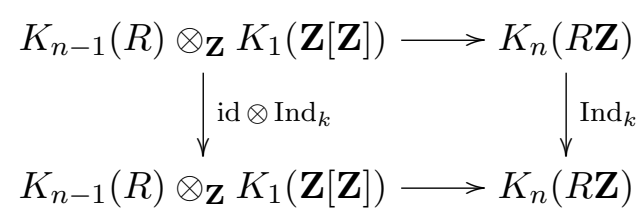

where the horizontal pairings are given by $\mu_{R, \mathbf{Z}}$ from (4.6). Since in $K_{1}(\mathbf{Z}[\mathbf{Z}]), k$ times the class $[t]$ of the unit $t$ is the class $\left[t^{k}\right]=\operatorname{Ind}_{k}([t])$, the claim follows for $K_{n-1}(R)$.

The commutativity when restricted to the copies of $\mathrm{NK}_{n}(R)$ follows from Lemma 2.9. This finishes the proof of Lemma 4.5.

Lemma 4.5 implies that the composite (4.4) and hence the composite (4.3) agree with the composite

$$
\begin{aligned}
K_{n}(R[P \times \mathbf{Z}]) \stackrel{r_{n}}{\longrightarrow} \mathrm{NK}_{n}(R P) \stackrel{\phi}{\rightarrow} \mathrm{NK}_{n}(R[P \times \mathbf{Z} / \ell]) & \stackrel{j_{*}}{\longrightarrow} \mathrm{NK}_{n}(R G) \stackrel{V_{k}}{\longrightarrow} \mathrm{NK}_{n}(R G) .
\end{aligned}
$$


Since we have already shown that the element $x \in \mathrm{NK}_{n}(R G)_{(p)}$ lies in the image of (4.2), we conclude that $x$ lies in the image of the map

$$
\Phi=\left(\Phi_{P}\right): \bigoplus_{P \in \mathfrak{P}_{p}(G)} \mathrm{NK}_{n}(R P)_{(p)} \rightarrow \mathrm{NK}_{n}(R G)_{(p)}
$$

subject only to the restriction $k \in I(g)$ in the definition of $\Phi_{P}$.

Consider $k \geq 1, P \in \mathfrak{P}_{p}$ and $g \in C_{G}^{\perp} P_{p}$. We write $k=k_{0} k_{1}$ for $k_{1} \in I(g)$ and $\left(k_{0},|g|\right)=1$. We have $V_{k}=V_{k_{1}} \circ V_{k_{0}}$ (see Stienstra [23, Theorem 2.12]). Since $\left(k_{0},|g|\right)=1$, we can find an integer $l_{0}$ such that $\left(l_{0},|g|\right)=1$ and $\left(g^{l_{0}}\right)^{k_{0}}=g$. We conclude from Stienstra [23, page 67]

$$
V_{k_{0}} \circ \phi(P, g)=V_{k_{0}} \circ \phi\left(P,\left(g^{l_{0}}\right)^{k_{0}}\right)=\phi\left(P, g^{l_{0}}\right) \circ V_{k_{0}} .
$$

Hence the image of $V_{k} \circ \phi(P, g)$ is contained in the image of $V_{k_{1}} \circ \phi\left(P, g^{l_{0}}\right)$ and $g^{l_{0}} \in C_{G}^{\perp} P_{p}$. This finishes the proof of Theorem A.

\section{EXAMPLES}

We briefly discuss some examples. As usual, $p$ is a prime and $G$ is a finite group. The first example shows that Theorem A gives some information about p-elementary groups.

Example 5.1. Let $P$ be a finite $p$-group and let $\ell \geq 1$ an integer with $(\ell, p)=1$. Then Theorem A says that $\mathrm{NK}_{n}(R[P \times \mathbf{Z} / \ell])_{(p)}$ is generated by the images of the maps

$$
V_{k} \circ \phi(P, g): \mathrm{NK}_{n}(R P)_{(p)} \rightarrow \mathrm{NK}_{n}(R[P \times \mathbf{Z} / \ell])_{(p)}
$$

for all $g \in \mathbf{Z} / \ell$, and all $k \in I(g)$. Since the composite $F_{k} \circ V_{k}=k \cdot$ id for $k \geq 1$ (see [23, Theorem 2.12]) and $(k, p)=1$, the map

$$
V_{k}: \mathrm{NK}_{n}(R G)_{(p)} \rightarrow \mathrm{NK}_{n}(R G)_{(p)}
$$

is injective for all $k \in I(g)$. For $g=1, \phi(P, 1)$ is the map induced by the first factor inclusion $P \rightarrow P \times \mathbf{Z} / \ell$, and this is a split injection. In addition, the composition of $\phi(P, g)$ with the functor induced by the projection $P \times \mathbf{Z} / \ell \rightarrow P$ is the identity on $\operatorname{NIL}(R P)$, for any $g \in \mathbf{Z} / \ell$. Therefore, all the maps $V_{k} \circ \phi(P, g)$ are split injective. It would be interesting to understand better the images of these maps as $k$ and $g$ vary. For example, what is the image of $\Phi_{\{1\}}$ where we take $P=\{1\}$ ? 
In some situations the Verschiebung homomorphisms and the homomorphisms $\phi(P, g)$ for $g \neq 1$ do not occur.

Example 5.2. Suppose that $R$ is a regular ring. We consider the special situation where the $p$-Sylow subgroup $G_{p}$ is a normal subgroup of $G$, and furthermore where $C_{G}(P) \subseteq P$ holds for every non-trivial subgroup $P \subseteq G_{p}$. For $P \neq\{1\}$, we have $C_{G}^{\perp}(P)=\{1\}$ and the homomorphism $\Phi_{P}=\phi(P, 1)$, which is the ordinary induction map. We can ignore the map $\Phi_{\{1\}}$ since $\mathrm{NK}_{n}(R)=0$ by assumption. Therefore, the (surjective) image of $\Phi$ in Theorem A is just the image of the induction map

$$
\mathrm{NK}_{n}\left(R G_{p}\right)_{(p)} \rightarrow \mathrm{NK}_{n}(R G)_{(p)}
$$

Note that $\mathrm{NK}_{n}\left(R G_{p}\right)$ is $p$-local, and we can divide out the conjugation action on $\mathrm{NK}_{n}\left(R G_{p}\right)$ because inner automorphisms act as the identity on $\mathrm{NK}_{n}(R G)$. However, $G / G_{p}$ is a finite group of order prime to $p$, so that

$$
H_{0}\left(G / G_{p} ; \mathrm{NK}_{n}\left(R G_{p}\right)\right)=H^{0}\left(G / G_{p} ; \mathrm{NK}_{n}\left(R G_{p}\right)\right)=\mathrm{NK}_{n}\left(R G_{p}\right)^{G / G_{p}} .
$$

Hence the induction map on this fixed submodule

$$
\lambda_{n}: \mathrm{NK}_{n}\left(R G_{p}\right)^{G / G_{p}} \rightarrow \mathrm{NK}_{n}(R G)_{(p)}
$$

is surjective. An easy application of the double coset formula shows that the composition of $\lambda_{n}$ with the restriction map $\operatorname{Res}_{G}^{G_{p}}: \mathrm{NK}_{n}(R G)_{(p)} \rightarrow \mathrm{NK}_{n}\left(R G_{p}\right)_{(p)}$ is given by $\left|G / G_{p}\right|$-times the inclusion $\mathrm{NK}_{n}\left(R G_{p}\right)^{G / G_{p}} \rightarrow \mathrm{NK}_{n}\left(R G_{p}\right)$. Since $\left(\left|G / G_{p}\right|, p\right)=1$ this composition, and hence $\lambda_{n}$, are both injective. We conclude that $\lambda_{n}$ is an isomorphism.

Concrete examples are provided by semi-direct products $G=P \rtimes C$, where $P$ is a cyclic $p$-group, $C$ has order prime to $p$, and the action map $\alpha: C \rightarrow \operatorname{Aut}(P)$ is injective. If we assume, in addition, that the order of $C$ is square-free, then

$$
\mathrm{NK}_{n}(\mathbf{Z} P)^{C} \stackrel{\cong}{\rightrightarrows} \mathrm{NK}_{n}(\mathbf{Z}[P \rtimes C])
$$

for all $n \leq 1$ (this dimension restriction, and setting $R=\mathbf{Z}$, are only needed to apply Bass-Murthy [4] in order to eliminate possible torsion in the Nil-group of orders prime to $p$ ). 


\section{6. $N K_{n}(A)$ AS A MACKEY FUNCTOR}

Let $G$ be a finite group. We want to show that the natural maps

$$
i_{n}: \mathrm{NK}_{n}(R G) \rightarrow K_{n}(R[G \times \mathbf{Z}])
$$

and

$$
r_{n}: K_{n}(R[G \times \mathbf{Z}]) \rightarrow \mathrm{NK}_{n}(R G)
$$

in the Bass-Heller-Swan isomorphism are maps of Mackey functors (defined on subgroups of $G)$. Hence $\mathrm{NK}_{n}(R G)$ is a direct summand of $K_{n}(R[G \times \mathbf{Z}])$ as a Mackey functor. Since $R G$ is a finitely generated free $R H$-module, for any subgroup $H \subset G$, it is enough to apply the following lemma to $A=R H$ and $B=R G$.

Lemma 6.1. Let $i: A \rightarrow B$ be an inclusion of rings. Then the following diagram commutes

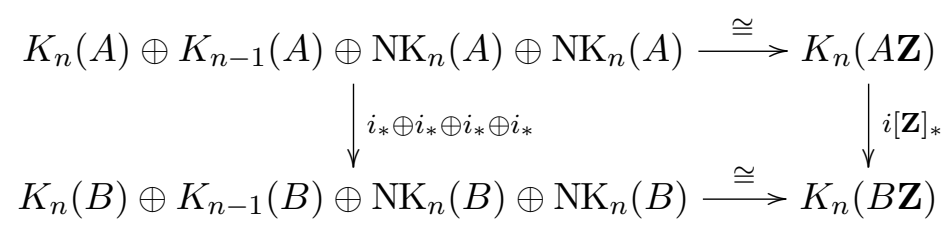

where the vertical maps are given by induction, and the horizontal maps are the Bass-Heller-Swan isomorphisms. If B is finitely generated and projective, considered as an A-module, then

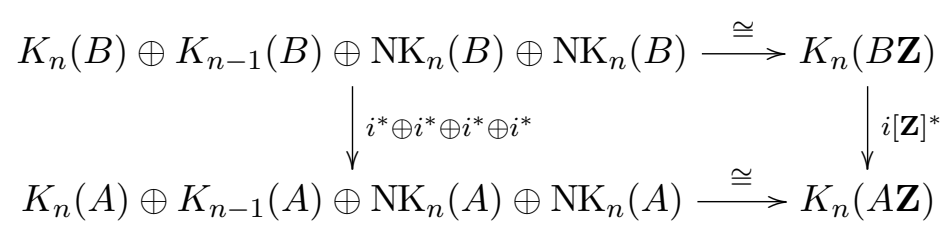

where the vertical maps are given by restriction, and the horizontal maps are the Bass-Heller-Swan isomorphisms.

Proof. One has to show the commutativity of the diagram when restricted to each of the four summands in the left upper corner. In each case these maps are induced by functors, and one shows that the two corresponding composites of functors are naturally equivalent. Hence the two composites induce the same map on $K$-theory. As an illustration we do this in two cases. 
Consider the third summand $\mathrm{NK}_{n}(A)$ in the first diagram. The Bass-HellerSwan isomorphism restricted to it is given by the the restriction of the map $\left(j_{+}\right)_{*}: K_{n}(A[t]) \rightarrow K_{n}\left(A\left[t, t^{-1}\right]\right)=K_{n}(A \mathbf{Z})$ induced by the obvious inclusion $j_{+}: A[t] \rightarrow A\left[t, t^{-1}\right]$ restricted to $\operatorname{NK}_{n}(A)=\operatorname{ker}\left(\epsilon_{*}: K_{n}(A[t]) \rightarrow K_{n}(A)\right)$, where $\epsilon: A[t] \rightarrow A$ is given by $t=0$. Since all these maps come from induction with ring homomorphisms, the following two diagrams commute

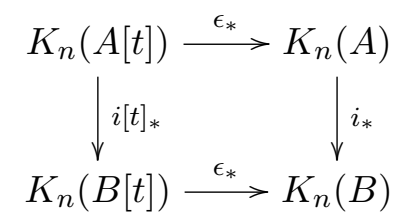

and

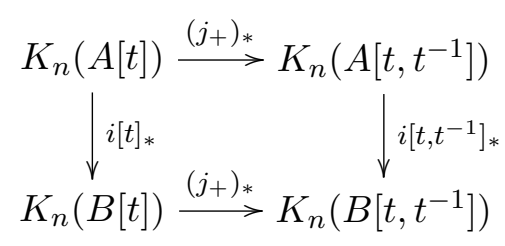

and the claim follows.

Consider the second summand $K_{n-1}(B)$ in the second diagram. The restriction of the Bass-Heller-Swan isomorphism to $K_{n-1}(B)$ is given by evaluating the pairing (4.6) for $\Gamma=\mathbf{Z}$ for the unit $t \in \mathbf{Z}[\mathbf{Z}]$. Hence it suffices to show that the following diagram commutes, where the horizontal maps are given by the pairing (4.6) for $\Gamma=\mathbf{Z}$ and the vertical maps come from restriction



This follows from the fact that for a finitely generated projective $A$-module $P$ and a finitely generated projective $\mathbf{Z}[\mathbf{Z}]$-module $Q$ there is a natural isomorphism of $B \mathbf{Z}$-modules

$$
\left(\operatorname{Res}_{A} P\right) \otimes_{\mathbf{Z}} Q \stackrel{\cong}{\rightarrow} \operatorname{Res}_{A \mathbf{Z}}\left(P \otimes_{\mathbf{Z}} Q\right), \quad p \otimes q \mapsto p \otimes q .
$$

Corollary 6.2. Let $G$ be a finite group, and $R$ be a ring. Then, for any subgroup $H \subset G$, the induction maps $\operatorname{Ind}_{H}^{G}: \mathrm{NK}_{n}(R H) \rightarrow \mathrm{NK}_{n}(R G)$ and the restriction maps $\operatorname{Res}_{G}^{H}: \operatorname{NK}_{n}(R G) \rightarrow \mathrm{NK}_{n}(R H)$ commute with the Verschiebung and Frobenius homomorphisms $V_{k}, F_{k}$, for $k \geq 1$. 
Proof. We combine the results of Lemma 6.1 with Stienstra's Lemma 2.9 (note that these two diagrams also commute with $i_{n}$ replaced by $r_{n}$ ).

\section{REFERENCES}

[1] A. Bartels and W. Lück, Isomorphism conjecture for homotopy K-theory and groups acting on trees, J. Pure Appl. Algebra 205 (2006), 660-696.

[2] H. Bass, Algebraic K-theory, W. A. Benjamin, Inc., New York-Amsterdam, 1968.

[3] H. Bass, A. Heller, and R. G. Swan, The Whitehead group of a polynomial extension, Inst. Hautes Études Sci. Publ. Math. (1964), 61-79.

[4] H. Bass and M. P. Murthy, Grothendieck groups and Picard groups of abelian group rings, Ann. of Math. (2) 86 (1967), 16-73.

[5] F. X. Connolly and M. O. M. da Silva, The groups $N^{r} K_{0}(\mathbf{Z} \pi)$ are finitely generated $\mathbf{Z}\left[\mathbf{N}^{r}\right]$ modules if $\pi$ is a finite group, $K$-Theory 9 (1995), 1-11.

[6] A. W. M. Dress, Contributions to the theory of induced representations, Algebraic $K$-theory, II: "Classical" algebraic $K$-theory and connections with arithmetic (Proc. Conf., Battelle Memorial Inst., Seattle, Wash., 1972), Springer, Berlin, 1973, pp. 183-240. Lecture Notes in Math., Vol. 342.

[7] _ Induction and structure theorems for orthogonal representations of finite groups, Ann. of Math. (2) 102 (1975), 291-325.

[8] F. T. Farrell, The nonfiniteness of Nil, Proc. Amer. Math. Soc. 65 (1977), 215-216.

[9] F. T. Farrell and W. C. Hsiang, Rational L-groups of Bieberbach groups, Comment. Math. Helv. 52 (1977), 89-109.

[10] _ The Whitehead group of poly-(finite or cyclic) groups, J. London Math. Soc. (2) 24 (1981), 308-324.

[11] J. Grunewald, The behaviour of Nil-groups under localization and the assembly map, Topology 47 (2008), 160-202.

[12] D. Grayson, Higher algebraic K-theory. II (after Daniel Quillen), Algebraic K-theory (Proc. Conf., Northwestern Univ., Evanston, Ill., 1976), Springer, Berlin, 1976, pp. 217-240. Lecture Notes in Math., Vol. 551.

[13] D. R. Grayson, The K-theory of endomorphisms, J. Algebra 48 (1977), 439-446.

[14] I. Hambleton, Some examples of free actions on products of spheres, Topology 45 (2006), 735-749.

[15] I. Hambleton and E. K. Pedersen, Identifying assembly maps in $K$ - and L-theory, Math. Ann. 328 (2004), 27-57.

[16] I. Hambleton, L. Taylor, and E. B. Williams, Dress induction and the Burnside quotient Green ring, Algebra \& Number Theory 3 (2009), 511-541.

[17] D. R. Harmon, $N K_{1}$ of finite groups, Proc. Amer. Math. Soc. 100 (1987), 229-232.

[18] E. K. Pedersen and C. A. Weibel, K-theory homology of spaces, Algebraic topology (Arcata, CA, 1986), Lecture Notes in Math., vol. 1370, Springer, Berlin, 1989, pp. 346-361. 
[19] D. Quillen, Higher algebraic K-theory. I, Algebraic $K$-theory, I: Higher $K$-theories (Proc. Conf., Battelle Memorial Inst., Seattle, Wash., 1972), Springer, Berlin, 1973, pp. 85-147. Lecture Notes in Math., Vol. 341.

[20] F. Quinn, Hyperelementary assembly for K-theory of virtually abelian groups, (arXiv:0509294v4[math.KT]), 2006.

[21] J. Rosenberg, Algebraic K-theory and its applications, Graduate Texts in Mathematics, vol. 147, Springer-Verlag, New York, 1994.

[22] V. Srinivas, Algebraic K-theory, second ed., Progress in Mathematics, vol. 90, Birkhäuser Boston Inc., Boston, MA, 1996.

[23] J. Stienstra, Operations in the higher $K$-theory of endomorphisms, Current trends in algebraic topology, Part 1 (London, Ont., 1981), CMS Conf. Proc., vol. 2, Amer. Math. Soc., Providence, R.I., 1982, pp. 59-115.

[24] R. G. Swan, Higher algebraic K-theory, K-theory and algebraic geometry: connections with quadratic forms and division algebras (Santa Barbara, CA, 1992), Proc. Sympos. Pure Math., vol. 58, Amer. Math. Soc., Providence, RI, 1995, pp. 247-293.

[25] J. B. Wagoner, Delooping classifying spaces in algebraic K-theory, Topology 11 (1972), 349-370.

[26] F. Waldhausen, Algebraic K-theory of spaces, Algebraic and geometric topology (New Brunswick, N.J., 1983), Lecture Notes in Math., vol. 1126, Springer, Berlin, 1985, pp. 318419.

[27] C. A. Weibel, Mayer-Vietoris sequences and module structures on $N K_{*}$, Algebraic $K$ theory, Evanston 1980 (Proc. Conf., Northwestern Univ., Evanston, Ill., 1980), Lecture Notes in Math., vol. 854, Springer, Berlin, 1981, pp. 466-493.

Ian Hambleton

Department of Mathematics \& Statistics

McMaster University

Hamilton, ON L8S 4K1, Canada.

Email: ian@math.mcmaster.ca

Wolfgang Lück

Mathematisches Institut

Universität Münster

D-48149 Münster, Germany.

Email: lueck@math.uni-muenster.de 